\title{
2883 SEPARATION OF PRIMARIES AND MULTIPLES BY NON-LINEAR ESTIMATION IN THE CURVELET DOMAIN
}

FELIX J. HERRMANN ${ }^{1}$ and ERIC VERSCHUUR ${ }^{2}$

${ }^{1}$ Department of Earth and Ocean Sciences, University of British Columbia, Canada

${ }^{2}$ Faculty of Applied Sciences, Delft University of Technology, The Netherlands

\begin{abstract}
Predictive multiple suppression methods consist of two main steps: a prediction step, in which multiples are predicted from the seismic data, and a subtraction step, in which the predicted multiples are matched with the true multiples in the data. The last step appears crucial in practice: an incorrect adaptive subtraction method will cause multiples to be sub-optimally subtracted or primaries being distorted, or both. Therefore, we propose a new domain for separation of primaries and multiples via the Curvelet transform. This transform maps the data into almost orthogonal localized events with a directional and spatial-temporal component. The multiples are suppressed by thresholding the input data at those Curvelet components where the predicted multiples have large amplitudes. In this way the more traditional filtering of predicted multiples to fit the input data is avoided. An initial field data example shows a considerable improvement in multiple suppression.
\end{abstract}

\section{Introduction}

In complex areas move-out filtering multiple suppression techniques may fail because underlying assumptions are not met. Several attempts have been made to address this problem by either extending move-out discrimination methods towards 3D complexities (e.g. by introducing apex-shifted hyperbolic transforms [7]) or by coming up with matching techniques in the wave-equation based predictive methods [see e.g. 17, 1]. Least-squares matching the predicted multiples in time and space overlapping windows, [16] provides a straightforward subtraction method, where the predicted multiples are matched to the true multiples for 2-D input data. Unfortunately, this matching procedure fails when the underlying 2D assumption are severely violated. There have been several attempts to address this issue and the proposed solutions range from including surrounding shot positions [11] to methods based on model- [14], data-driven [10] time delays and separation of predicted multiples into (in)-coherent parts [12]. Even though these recent advances in adaptive subtraction and other techniques have improved the attenuation of multiples, these methods continue to suffer from (i) a relative strong sensitivity to the accuracy of the predicted multiples; (ii) creation of spurious artifacts or worse (iii) a possible distortions of the primary energy. For these situations, subtraction techniques based on a different concept are needed to complement the processor's tool box.

The method we are proposing here holds the middle between two complementary approaches common in multiple elimination: prediction in combination with subtraction and filtering [15, 7]. Whereas the first approach aims to predict the multiples and then subtract, the second approach tries to find a domain in which the primaries and multiples separate, followed by some filtering operation and reconstruction. Our method is not distant from either since it uses the predicted multiples to non-linearly filter data in a domain spanned by almost orthogonal and local basis functions. We use the recently developed Curvelet transform [see e.g. 3], that decomposes data into basis functions that not only obtain optimal sparseness on the coefficients and hence reduce the dimensionality of the problem but which are also local in both location and angle/dip, facilitating the definition of non-linear estimators based on thresholding. Main assumption of this proposal is that multiples and primaries have locally a different temporal, spatial and dip behavior, and therefore map into different areas in the Curvelet domain. Multiples give rise to large Curvelet coefficients in the input and these coefficients can be muted by our estimation procedure when the threshold is set according to the Curvelet transform of the predicted multiples. As such, our suppression technique has at each location in the transformed domain one parameter, namely the threshold yielded by the predicted multiple, beyond which the input data is suppressed. In that sense, our procedure is similar to the ones proposed by [18] and [15], although the latter use the non-localized FK/Radon domains for their separation while we use localized basis functions and non-linear estimation by thresholding. Non-locality and non-optimality in their approximation renders the first filtering techniques less effective because primaries and multiples will still have a considerable overlap. The Curvelet transform is able to make a local discrimination between interfering events with different temporal and spatial characteristics.

\section{Adaptive subtraction by non-linear estimation of the Curvelet coefficients}

\section{The denoising problem}

Removing predicted multiples can be seen as a particular instance of a generic denoising problem that estimates the model (primaries) $\mathbf{m}$ from noisy data (data including multiples) [See e.g. 13]

$$
\mathbf{d}=\mathbf{m}+\mathbf{n}
$$


with $\mathbf{n}$ white Gaussian noise. Main difference between this problem and ours is that in our case the noise is coherent and considered to be the multiples to which we have access through prediction.

Question now is: how can we solve this denoising problem effectively? In other words, how can we construct a diagonal decision operator that minimizes the energy difference between the estimate and the true model. It appears from the work of [6], [13] and others that, for a certain class of models, one can obtain nearly optimal denoising results, i.e. near optimal SNR for denoised data, by projecting noisy data onto a basis-function representation that is optimal for that particular class of models. In that case, most of the model's energy resides in only a few coefficients, allowing for the definition of a shrinkage estimator that separates noise from the model. For basis functions that are also local, one can show that soft thresholding on the coefficients suffices to approximately solve the denoising problem, i.e.

$$
\hat{\mathbf{m}}=\mathbf{B}^{-1} \theta_{\mu}(\mathbf{B d}) \text {. }
$$

In this expression, $\mathbf{B}^{-\mathbf{1}}$ refers to the (pseudo)-inverse of $\mathbf{B}$, which is the basis-function expansion. $\theta_{\mu}$ is a soft/hard thresholding operator with a threshold that for orthonormal basis functions equals [13, 6] $\mu=\sigma \sqrt{2 \log _{e} N}$ with $\sigma$ the standard deviation of the noise and $N$ the number of data samples. Question now is: can we extend these results to colored noise and to be specific to noise given by the predicted multiples? Before answering this question let us first be more specific with respect to the choice of the appropriate basis functions for seismic data, primaries as well as multiples.

\section{The basis functions}

Curvelets as proposed by [3], constitute a relatively new family of non-separable wavelet bases that are designed to effectively represent seismic data with reflectors that generally tend to lie on piece-wise smooth curves. This property makes Curvelets suitable to represent events in seismic whether these are located in shot records or time slices. For these type of signals, Curvelets obtain nearly optimal sparseness, because of (i) the rapid decay for the reconstruction error as a function of the largest coefficients; (ii) the ability to concentrate the signal's energy in a limited number of coefficients; (iii) the ability to map noise and signal to different areas in the Curvelet domain. So how do Curvelets obtain such a high non-linear approximation rate? Without being all inclusive [see for details 2, 4, 3, 5], the answer to this question lies in the fact that Curvelets are

- multi-scale, i.e. they live in different dyadic corona (see for more detail [3] or the other contributions of the fist author to the proceedings of this conference) in the FK-domain.

- multi-directional, i.e. they live on wedges within these corona.

- anisotropic, i.e. they obey the following scaling law width $\propto$ length $^{2}$.

- directional selective with \# orientations $\propto \frac{1}{\sqrt{\text { scale }}}$.

- local both in $(x, t))$ and $\mathrm{KF}$.

- almost orthogonal, they are tight frames with a moderate redundancy.

Curvelets live in a wedges of the 2-D Fourier plane and become more directional selective and anisotropic for the higher frequencies. They are localized in both the space (or $(x, t)$ ) and spatial KF-domains and have, as consequence of their partitioning, the tendency to align themselves with curves/wavefronts. As such they are more flexible then a representation yielded by highresolution Radon [as described by e.g. 15] because they are local and able to follow any piece-wise smooth curve.

\section{A tantalizing perspective}

As the examples in the next section clearly demonstrate, the optimal denoising capabilities for incoherent noise carry over to coherent noise removal provided we have reasonable accurate predictions for the noise, the multiples in this case. By choosing a threshold defined by the predicted multiples, i.e.

$$
\mu \sim \eta|\mathbf{B n}|
$$

we are able to awe are able to adaptively decide whether a certain event belongs to primary or multiple energy. The $\mathbf{n}$ contains the predicted multiples and $\eta$ represents an additional control parameter which sets the confidence interval (e.g. $95 \%$ for $\eta=3$ ) (de)-emphasizing the thresholding.

\section{Results of multiple subtraction via thresholding in the Curvelet domain}

The above methodology is tested on a field dataset from offshore Scotland. This is a 2D line, which is known to suffer from strong surface-related multiples. Because the geology of the shallow sub-bottom layers is laterally complex, multiples have been observed to exhibit 3D characteristics. Applying surface-related multiple elimination to this data gives only an attenuation of surface multiples, but not a complete suppression. This lack of suppression can be observed for a shot record shown in Fig. 1 When comparing the predicted multiples with the input data a good resemblance is observed in a global sense. However, when a matching filter is calculated, it appears that the predicted multiples do not coincide well enough with the true multiples, mainly due to $3 \mathrm{D}$ effects. Locally the temporal and spatial shape of the predicted multiples - especially for the higher order multiples - differs from the true events. Estimating shaping filters in spatially and temporally varying, overlapping windows, as described by [16] can not resolve this mismatch completely. The adaptive subtraction result is displayed in Fig. 11 in the third panel. To improve this result, more freedom can be incorporated in the subtraction process. However, note that the more the predicted multiples can adapt to the input data, the higher the chance that primary energy will be distorted as well, as the primary and multiple energy are not orthogonal to each other in the least-squares sense. Multi-gather subtraction using 9 surrounding multiple panels gives better suppression of the multiples but leaves a lot of multiple remnants to be observed, especially for the large offset at large travel times. The output gather of the Curvelet method (see fourth panel in Fig. 1p looks much cleaner. Furthermore, clear events have been restored from interference with the multiples in the lower left area. Also note the good preservation of a primary event around -1000 meter offset 

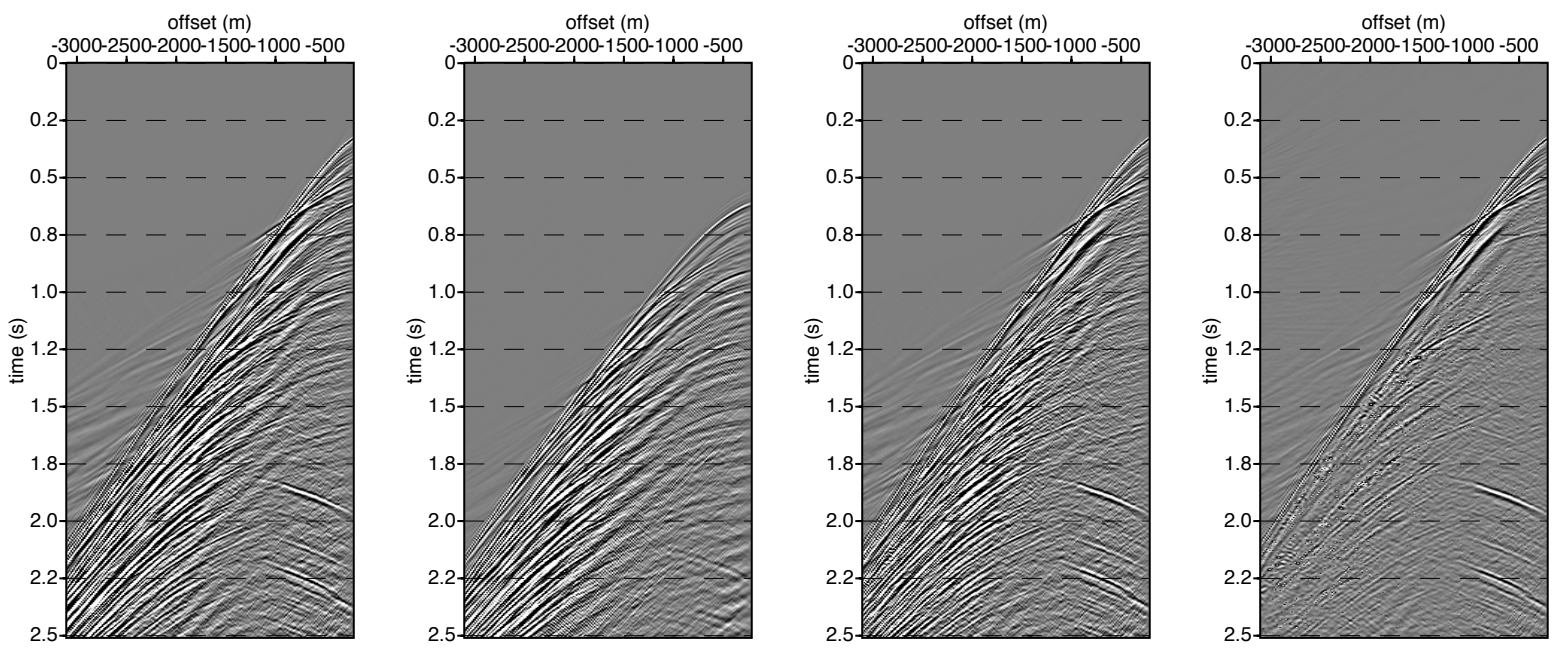

Figure 1: Comparison between adaptive least-squares (using time and space varying least-squares filters)and Curvelet subtraction of predicted multiples for one shot record. Input data (left), predicted multiples (second), least-squares subtraction (third) and the results by thresholding Curvelet (right).

and 1.1 seconds. The amplitudes seem to be identical to the ones in the input data, whereas the adaptive subtraction has distorted these amplitudes to some extend. For the Curvelet-domain procedure, the threshold value can be set by the user, thus creating more or less suppression. Too weak thresholding leaves too much multiples in the data and that a too strong thresholding procedure seems to remove too much energy.

The filtering via the Curvelet domain can also be applied within each 2D cross-section through the seismic data volume. Time slices at $t=1.48$ through the prestack shot-offset data and predicted-multiple volumes are displayed in Fig. 2 (top row). Results after application of the adaptive subtraction per shot record and the time-slice only Curvelet subtraction are included in Fig. 2 (bottom row). Again, the Curvelet results look much cleaner than the least-squares subtraction result. Much of the noisy remnants at small offsets in the least-squares subtraction result have been removed. Notice also the improved suppression of the higher order peg-legs in the area around shot 900 and offset 1500 meter.

\section{Conclusions}

In this paper a new concept related to multiple subtraction has been described, based on the Curvelet transform. The Curvelet transform is an almost orthogonal transformation into local basis functions parameterized by their relate temporal- and spatialfrequency content. Because of their anisotropic shape, Curvelets are directional selective, i.e. they have local angle-discrimination capabilities. Our method uses the predicted multiples from the surface-related multiple prediction method, as a guide to suppress the Curvelet coefficients related to the multiple events directly in the original data. Therefore, the multiples are not actually subtracted, but areas in the Curvelet domain related to multiple energy are muted. The success of this method depends on the assumption that primaries and multiples map into different areas in the Curvelet domain. Based on a field data example, we can conclude that the Curvelet-based multiple filtering is effective and is able to suppress multiples, while preserving primary energy. Especially in situations with clear 3D effects in the 2D seismic data, it appears to perform better than the more traditional least-squares adaptive subtraction methods. This success does not really come as a surprise given the successful application of these techniques to the removal of noise colored by migration [8, 9] and to the computation of 4D difference cubes (see for details in both other contributions of the first to proceedings of this conference). Leaves us to hope for future higher dimensional implementation of the Curvelet transform possibly supplemented by constraint optimization imposing sparseness constraints (see the migration paper in these proceedings).

\section{Acknowledgments}

The authors thank Elf Caledonia Ltd (now part of Total) for providing the field data and Emmanuel Candés and David Donoho for making an early version of their Curvelet code (Digital Curvelet Transforms via Unequispaced Fourier Transforms, presented at the ONR Meeting, University of Minnesota, May, 2003) available for evaluation. This work was in part financially supported by a NSERC Discovery Grant.

\section{References}

[1] A. J. Berkhout and D. J. Verschuur. Estimation of multiple scattering by iterative inversion, part I: theoretical considerations. Geophysics, 62(5):1586-1595, 1997.

[2] E. J. Candès and D. L. Donoho. Curvelets - a surprisingly effective nonadaptive representation for objects with edges. Curves and Surfaces. Vanderbilt University Press, 2000. 

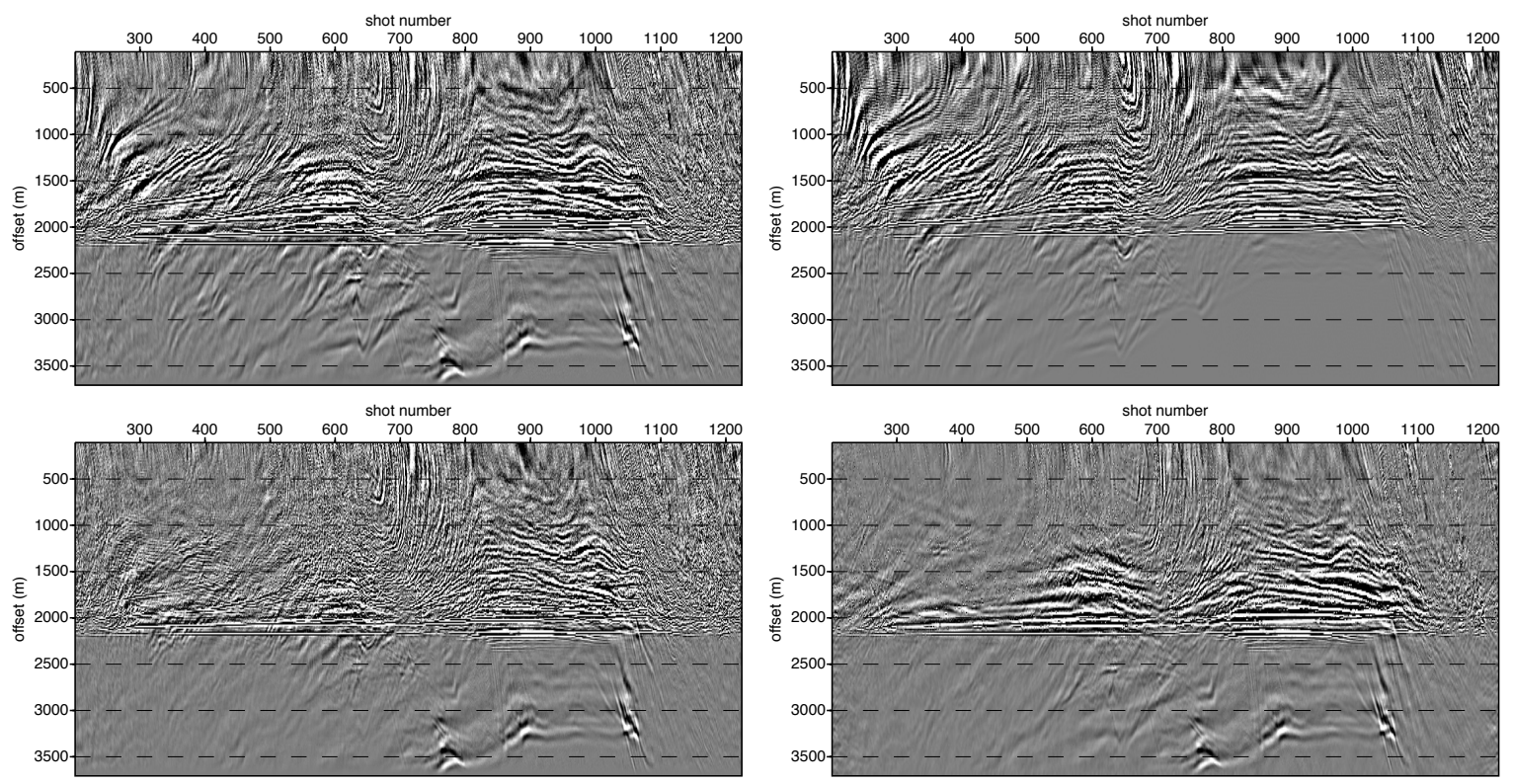

Figure 2: Time slice through the prestack data volume of all shot records. Top left: Time slice through the input data with all multiples. Top right: Slice through the 2D predicted surface-related multiples. Bottom left: Time slice through the shot records after adaptive subtraction per shot record of the predicted multiples. Bottom right: The Curvelet equivalent of the adaptive subtraction computed for a single time horizon.

[3] E. J. Candès and F. Guo. New multiscale transforms, minimum total variation synthesis: Applications to edge-preserving image reconstruction. Signal Processing, pages 1519-1543, 2002.

[4] E. J. E. J. Candès and D. Donoho. New tight frames of curvelets and optimal representations of objects with smooth singularities. Technical report, Stanford, 2002. submitted.

[5] M. Do and M. Vetterli. Beyond wavelets, chapter Contourlets. Academic Press, 2002.

[6] D. L. Donoho and I. M. Johnstone. Minimax estimation via wavelet shrinkage. Annals of Statistics, 26(3):879-921, 1998.

[7] N. Hargreaves, B. verWest, R. Wombell, and D. Trad. Multiple attenuation using apex-shifted radon transform. pages 1929-1932, Dallas, 2003. SEG, Soc. Expl. Geophys., Expanded abstracts.

[8] F. J. Herrmann. Multifractional splines: application to seismic imaging. In A. F. L. E. Michael A. Unser, Akram Aldroubi, editor, Proceedings of SPIE Technical Conference on Wavelets: Applications in Signal and Image Processing X, volume 5207, pages 240-258. SPIE, 2003.

[9] F. J. Herrmann. Optimal seismic imaging with curvelets. In Expanded Abstracts, Tulsa, 2003. Soc. Expl. Geophys.

[10] L. T. Ikelle and S. Yoo. An analysis of 2D and 3D inverse scattering multiple attenuation. pages 1973-1976, Calgary, 2000. SEG, Soc. Expl. Geophys., Expanded abstracts.

[11] H. Jakubowicz. Extended subtraction of multiples. Private communication, Delft, 1999.

[12] M. M. N. Kabir. Weighted subtraction for diffracted multiple attenuation. pages 1941-1944, Dallas, 2003. SEG, Soc. Expl. Geophys., Expanded abstracts.

[13] S. G. Mallat. A wavelet tour of signal processing. Academic Press, 1997.

[14] W. S. Ross. Multiple suppression: beyond 2-D. part I: theory. Delft Univ. Tech., pages 1387-1390, Dallas, 1997. Soc. Expl. Geophys., Expanded abstracts.

[15] D. O. Trad. Interpolation and multiple attenuation with migration operators. Geophysics, 68(6):2043-2054, 2003.

[16] D. J. Verschuur and A. J. Berkhout. Estimation of multiple scattering by iterative inversion, part II: practical aspects and examples. Geophysics, 62(5):1596-1611, 1997.

[17] D. J. Verschuur, A. J. Berkhout, and C. P. A. Wapenaar. Adaptive surface-related multiple elimination. Geophysics, 57(9):1166-1177, 1992.

[18] B. Zhou and S. A. Greenhalgh. Wave-equation extrapolation-based multiple attenuation: 2-d filtering in the f-k domain. Geophysics, 59(10):1377-1391, 1994 